\title{
EL TRÁFICO DE INFLUENCIAS EN LA ESPAÑA FRANQUISTA: DECISIONES PÚBLICAS, BENEFICIOS PRIVADOS
}

\author{
Francisco Javier Fernández Roca* \\ Universidad Pablo de Olavide, Sevilla, España, <jfernandez@upo.es>
}

Resumen. En la España del primer franquismo, la política económica autárquica y el marco institucional intervencionista provocaron que los agentes económicos no se comportaran buscando la eficiencia económica, sino como buscadores de rentas. En este artículo se estudia, en primer lugar, el tráfico de influencias en las entregas directas de activos o en las asignaciones sesgadas de bienes, legitimados de alguna forma, por parte del poder público; en segundo lugar, se analiza cómo operaron las estructuras administrativas y burocráticas del Estado. Finalmente, se estudia la generación de beneficios particulares obtenidos desde los programas económicos y desde las normativas de la política económica.

Palabras clave: Corrupción, franquismo, riqueza, burocracia, dictadura, industria textil.

Abstract. During the first regime of the Franco dictatorship, the economic policy implemented in Spain was autarky. This policy and the high level of government interventionism lead the economic agents to behave as rent-seekers instead of seeking efficiency. In this paper, we firstly study the peddling of political favors not only through direct deliveries of assets but also trough the slanted assignments of goods. These two aspects were legitimized, in some way, by the State. Secondly, we analyze the role played by the government administrative and bureaucratic structures. Lastly, we study the private profits obtained from both economic programs and the regulations established by the economic policy.

Key words: Corruption, francoism, wealth, bureaucracy, dictatorship, textile industry.

Fecha de recepción: enero de 2011. Fecha de aceptación: junio de 2011

* El autor manifiesta su agradecimiento a los participantes de la sesión dedicada a la corrupción política en el II Congreso Latinoamericano de Historia Económica (CLADHE) por las sugerencias recibidas y a su colega Guadalupe Valera por la paciencia de leer varias versiones del manuscrito. Los errores sólo me incumben a mí. Igualmente, el autor agradece el financiamiento recibido del Ministerio de Innovación a través del proyecto 2010/0041/001 y de la Consejería de Economía e Innovación (Junta de Andalucía) a través del proyecto SEJ 4129.

Am. Lat. Hist. Econ., año 19, núm. 2 (38), mayo-agosto, 2012, pp. 193-218 


\begin{abstract}
Bastaba tener la influencia política necesaria para convertirse en un "empresario" de éxito. Resultaba empresarialmente más interesante "convencer" a algún político o a algún funcionario para que te concediera alguna licencia, que pensar en la innovación o la mejora de la empresa. ${ }^{1}$
\end{abstract}

\title{
INTRODUCCIÓN
}

$\int \sqrt{1}$ a historia económica de muchos países o, al menos, amplios periodos históricos de la misma, puede ser entendida tras la comprensión de los hechos de corrupción gestados en las instituciones estatales. ${ }^{2}$ Así, aunque en muchas ocasiones se habla de manera diferenciada de tráfico de influencias, de favores políticos a determinados agentes, de corruptelas político-económicas, en todos los casos se trata de alguna de las diferentes formas que adopta la corrupción en un determinado régimen político.

El concepto de corrupción se puede entender como el uso del poder público para lograr réditos privados mediante el abuso de poder, o de su ejercicio, por parte de la burocracia estatal. ${ }^{3}$ Andvig y Fjeldstad insisten en la importancia no sólo de la corrupción procedente de la violación de las normas legales, sino en la ruptura de la moral pública que lleva a actuar a los actores por cuestiones de relaciones de poder, amiguismo, favores debidos, etc. Es en esta línea de ruptura de la moral pública y de la prestación de favores para la consecución de beneficios privados en la que, en este trabajo, enmarco el tráfico de influencias. De este modo, el artículo no trata sobre delitos que se cometen por corrupción, que varían en el tiempo y las legislaciones, sino que se centra en algo más sutil: el tráfico de influencias; es decir, la ayuda -favores- suministrada desde el poder político o institucional a los agentes privados para la consecución de sus fines. Ayuda que se mueve, en muchas ocasiones, en una esfera de ilegalidad, de forzamiento de la legalidad vigente, o de reparto de favores discrecionales, y que no tiene por qué incluir el pago de sobornos.

En el caso concreto de España durante el primer franquismo, entre 1939 y 1959, se aúnan dos de las posibilidades enumeradas por Vitelli al catalogar las vías de corrupción. ${ }^{4}$ Por un lado, aquella proveniente de los instrumentos de la política económica y que habitualmente queda encubierta bajo las reglamentaciones y, por otro, la nacida dentro del aparato

\footnotetext{
${ }^{1}$ Barciela, "Franquismo", 1998, p. 91.

2 Sautu, Catálogo, 2004.

3 Andvig y Fjeldstad, "Research", 2000, p. 11.

${ }^{4}$ Vitelli, Corrupción, 2010, p. 3.
} 
burocrático del Estado y gestada por los funcionarios, o bien impulsada por agentes económicos que incitaban a los funcionarios. Siguiendo esta clasificación, en la España franquista hubo, en primer lugar, porcentajes elevados de corrupción en las entregas directas de activos o en las asignaciones sesgadas de bienes, legitimados de alguna forma, por parte del poder público a los agentes; en segundo lugar, en cómo operaron las estructuras administrativas y burocráticas del Estado y, en tercer lugar, en la generación de beneficios particulares obtenidos desde los programas económicos y desde las normativas de la política económica. ${ }^{5}$

$\mathrm{El}$ artículo tiene la siguiente estructura. Un primer apartado en el que se presenta una rápida mirada al marco institucional y burocrático en el que se desarrollaron las distintas formas de corrupción, tráfico de influencias, amiguismo y favores prestados. Un segundo apartado está destinado a explicar las vicisitudes que tuvo que enfrentar una empresa española de nueva creación -de las mayores por tamaño- para salir triunfante de las trabas puestas por el régimen. ${ }^{6}$ En concreto, se señala la importancia que el factor político desempeñó, superior al cálculo sobre la viabilidad económica, para denegar y, posteriormente, autorizar la instalación de la nueva fábrica, mostrándose cómo operaron las estructuras burocráticas del Estado. La última sección se centra en cómo las decisiones políticoadministrativas tuvieron una clara incidencia en los resultados económicos de las empresas, es decir, cómo las decisiones públicas determinaron los beneficios privados.

\section{EL MARCO INSTITUCIONAL ESPAÑOL DEL PRIMER FRANQUISMO}

En la bibliografía española pueden encontrarse suficientes explicaciones sobre las razones, la forma en la que se llevaron a cabo y los efectos que tuvieron para la economía del país las decisiones de política económica por las que los gobiernos franquistas implementaron una economía autárquica y un fuerte intervencionismo. ${ }^{7}$ La autarquía, para cumplir con los objetivos gubernamentales de acabar con el desequilibrio exterior y con la depen-

5 "La corrupción administrativa se presenta cuando los funcionarios públicos con capacidad de otorgar contratos privados como concesiones, adquisición de bienes, contratación de personal o asignaciones de subsidios, o autorizaciones para realizar actividades económicas, perciben dineros privados para sesgar su decisión.” Ibid., p. 6.

${ }^{6}$ HYTASA se mantuvo entre las 200 empresas industriales de mayor tamaño de España en sus 40 años de historia. Carreras y Tafunell, "Gran”, 1993.

${ }^{7}$ Clavera, Autarquía, 1973; Tamames, República, 1973; Donges, Industrialización, 1976; González, Economía, 1979, y "Autarquía”, 1989-1990; Gamir, Política, 1980; Buesa, "Restricciones", 1984; Braña, Buesa y Molero, "Fin”, 1979; Braña, Buesa, Molero, Estado, 1984; García, "Notas”, 1985, e "Industrialización”, 1987; Martín y Comín, INI, 1991; Myro, "Industria”, 1993; Allende, Empresarios, 
dencia económica de la nación por medio de una política de sustitución de importaciones y de promoción de la producción nacional, dificultó la relación entre la economía española y el mercado internacional. Por su parte, el intervencionismo dispuso de un marco institucional cuyas disposiciones legales le concedía al gobierno la distribución de las materias primas, el reparto de los permisos de instalación de nuevas empresas, la concesión de los permisos de ampliación de fábricas y la dirección del comercio exterior de un elevado número de productos mediante el comercio de Estado. ${ }^{8}$

El resultado de ambas políticas fue la sustitución de los mecanismos del mercado por decisiones administrativas que provocaron la distorsión en la asignación de los recursos, originando estrangulamientos en la disposición de materias primas y energía y limitaciones en la renovación de la maquinaria. Además, la viabilidad de las empresas dejó de depender de su eficiencia económica para trasladarse a la eficacia del empresario en la obtención de rentas políticas que encaminaran en su favor las decisiones administrativas. ${ }^{9}$ De este modo, la política económica del franquismo potenció actitudes empresariales improductivas al convertir a los empresarios en activos buscadores de rentas o rent-seeking. ${ }^{10}$ La discrecionalidad de la administración modificó el patrón de comportamiento de los agentes y consolidó actividades improductivas como la especulación, el tráfico de influencias y la corrupción. ${ }^{11}$

Las actuaciones de los empresarios en la estructura político-administrativa del régimen han llevado a algunos autores a hablar de la "captura" del Estado, cuando empresarios y los sindicatos verticales aprovecharan su renta de situación en favor de los intereses de los empresarios instalados. La posición que les permitiría tal captura provenía de una legislación que obligaba a un largo expediente burocrático en el que se incluía tanto los informes de los industriales del sector como el del sindicato vertical correspondiente, circunstancia que ambos aprovecharían para opinar de manera contraria a la apertura de nuevas factorías. ${ }^{12}$ Posturas contrarias -la del sindicato vertical del sector y la de los empresarios concernidos- sustentadas en los criterios del Ministerio de Industria que, dada la situación de la España de posguerra, eran bastante fáciles de invocar en un infor-

1995; Comín, Empresa, 1995, "Empresa”, 1996, Rasgos, 1996, y "Estado", 2003; Gómez, Mitos, 2000; Barciela et al., España, 2001, y Barciela, Autarquía, 2003.

${ }^{8}$ En consecuencia, fue el gobierno, no el mercado, quien asignó los recursos y no fueron los precios los que transmitieron la información a los agentes económicos, sino las disposiciones gubernativas. Comín, "Estado", 1993, p. 49.

${ }^{9}$ Cabrera, "Intereses", 1996, y Cabrera y Rey, "Condena", 1994.

${ }^{10}$ Alonso y Torres, "Funciones", 1995.

${ }^{11}$ Barciela, "Franquismo", 1998.

${ }^{12}$ Buesa, "Restricciones", 1984; Miranda, "Industria”, 1994, Industria, 1998, y "Fracaso", 2003. 
me que justificara la negativa a la entrada de nuevos competidores. ${ }^{13}$ Por ejemplo, en la industria del calzado se presentaron expedientes para más de un millar de nuevas empresas de las que apenas una cuarta parte logró sus objetivos frente a 50\% de solicitudes de fabricantes que consiguieron mejoras para las instalaciones ya en producción (permisos de inversión, instalación de maquinaria nueva, talleres, etc.). Y como concluía un empresario zapatero respecto a sus dificultades para saltar las barreras de entrada: estas (las barreras de entrada) estribaban en que a los industriales lo que les interesaba era cerrar el sector y llegar a un "modo de monopolio en beneficio de determinadas empresas". ${ }^{14}$

Sin embargo, Pires matiza dicha visión de la captura cuando señala que fue precisamente el carácter dictatorial del régimen el que hizo que la captura se alejara de lo anticipado por la teoría y que la toma de las decisiones sobre autorizaciones y denegaciones de nuevas industrias quedara controlada por unos pocos funcionarios y se realizara "sin ninguna garantía ni control legal", acentuándose el peso "de la influencia individual y la discrecionalidad". ${ }^{15}$ La conclusión es que, aunque hubo casos en los que los industriales lograron frenar la instalación de nuevas fábricas, en general los funcionarios del Ministerio de Industria gozaron de autonomía suficiente para sortear las presiones de los grupos de industriales.

Más allá del grado de captura del Estado, lo que la bibliografía ha puesto de manifiesto es la importancia que tuvo el factor político, durante el franquismo, en el discurrir de las empresas. Las decisiones políticas incidían decisivamente en la viabilidad o inviabilidad económica de las empresas y, por ello, los empresarios recurrieron a "sus contactos y relaciones personales con los responsables [...] a fin de solventar las trabas administrativas". ${ }^{16}$ A continuación se muestra cómo algunos empresarios encontraron los modos de sortear las barreras de entrada que el régimen instauró bajo el paraguas intervencionista y de las que se trataban de aprovechar las empresas instaladas.

${ }^{13}$ Los criterios (1942) que guiaban al ministerio para aprobar, o denegar, los expedientes de nueva instalación industrial fueron: a) utilización por las industrias de materias primas escasas; b) existencia en ciertas industrias de una capacidad de producción muy superior a la posibilidad de absorción de la demanda interna, y $c$ ) desarrollo de algunas producciones que provocara el encarecimiento de productos básicos para los hogares. Dirección General de Industria, Ministerio de Industria y Comercio, 1942, en Buesa, "Restricciones", 1984, pp. 109-110.

${ }^{14}$ Torres intenta hacer una estimación de los efectos empresariales para España. Véanse Torres, "Empresa", 2003, pp. 180-181, y Miranda, Industria, 1998, pp. 261-262.

${ }^{15}$ Pires, Regulación, 2003, y "Empresarios", 2005, p. 147.

${ }^{16}$ Torres, "Empresa", 2003, pp. 181-182. 


\section{EL FAVOR POLÍTICO: DE REFUERZO DE LA BARRERA DE ENTRADA}

\section{A PÉRTIGA PARA SALTARLA}

En esta sección se analiza cómo el condicionamiento industrial franquista y los diferentes actores que se movían en el marco institucional, construido por el régimen, influyeron de manera decisiva en el nacimiento y discurrir de una empresa. El ejemplo que ilustra esta idea es el de la empresa sevillana Hilaturas y Tejidos Andaluces, S. A. (HYTASA), que permite mostrar tanto el camino burocrático a través de la administración del primer franquismo como los actores políticos que jugaron en su favor y en su contra y concluir en la importancia de estos en el devenir de la empresa.

HYTASA se fundó en Sevilla durante la guerra civil de 1937, con un capital de 10000000 de pesetas, cuando el conflicto eliminaba el problema de la competencia de los textiles catalanes -geográficamente localizados en el bando republicano-, aprovechándose del impulso que recibió el cultivo algodonero durante la dictadura del general Primo de Rivera y sustentándose sobre la integración vertical de la producción. ${ }^{17}$

En plena guerra civil, el primer movimiento realizado para poner en marcha la fábrica fue el de granjearse la amistad y connivencia de la máxima autoridad en el territorio, el general Queipo de Llano, jefe de los ejércitos del frente sur y figura emblemática de la rebelión militar. La obtención del favor político de Queipo tuvo unos inicios titubeantes que dieron con los fundadores de HYTASA, Prudencio Pumar y Luis Cobián, en la cárcel. ${ }^{18}$ Sin embargo, despejado el malentendido que provocó el encarcelamiento -una supuesta perversa utilización del nombre del general como patrocinador de la fábrica-, Queipo de Llano se convirtió en el principal garante de HYTASA y puso en juego todo su poder e influencia en su favor. El primer éxito de los fundadores se había alcanzado con el apoyo expreso del poder político hacia el proyecto.

El siguiente paso era poner en marcha el procedimiento administrativo. El proceso burocrático se resume en: a) elevar una memoria detallada del plan previsto al Ministerio de Industria en la que se solicitaban los permisos pertinentes; b) la publicación en el diario oficial para generar conocimiento del permiso solicitado; $c$ ) los escritos de oposición a la iniciativa, de empresarios privados o agentes institucionales; $d$ ) el pliego de descargo de los solicitantes, y $e$ ) la toma de decisión por parte de la autoridad previa consulta a sindicatos y delegaciones. El procedimiento burocrático

${ }^{17}$ La provincia de Sevilla acumuló la mayor formación de sociedades anónimas en 1937 con un total de 16, las cuales escrituraron 45900000 de pesetas de capital de los que HYTASA representaba 20\%. Véase Catalan, "Guerra", 2006, p. 195.

18 "Yo me quedé aterrado, porque por aquella época las detenciones solían tener malas consecuencias, y muchas iban seguidas de fusilamiento." Pumar, Tres, 1974, p. 141. 
finalizaba con la decisión de la autoridad política, la cual no siempre era plenamente firme, pues podía variar cuando se producían relevos en los altos cargos de la administración.

Una vez ganado el apoyo del general Queipo, los gestores de HYTASA pidieron la imprescindible autorización a la Junta Técnica del Estado (presidida por el general Gómez Jordana) para la constitución legal de la sociedad, para la instalación de la fábrica de hilados y tejidos de algodón y, por último, la autorización para la importación de la maquinaria extranjera. Aunque confiaban en que por medio de Queipo todo se podría solventar sin mayores trabas, conseguir los permisos supuso un enorme esfuerzo para los fundadores. Las dificultades aparecieron inmediatamente ya que la Comisión de Industria, Comercio y Abastos de la Junta Técnica retrasó su dictamen más de dos meses, según Pumar porque en ella "existían señores que no acogieron con buenos ojos nuestro proyecto y que estuvieron dispuestos a hacerlo fracasar". ${ }^{19}$ ¿Quiénes se oponían a la construcción de HYTASA? Las sospechas recaen sobre los grupos de presión catalanes que actuaban cerca del gobierno de Burgos desde fechas muy tempranas y que tendrían en el también catalán Joaquín Bau Nolla, presidente de la Comisión de Industria y Comercio, un buen apoyo. En octubre de 1936 casi un centenar de relevantes personalidades catalanas declaraban su adhesión al movimiento. ${ }^{20}$ Sabemos de patronales catalanas que estaban plenamente activas en 1939, así el Gremio de Fabricantes de Sabadell, el Instituto Tecnológico de Tarrasa y la agrupación de Hiladores de Barcelona tenían destacada a una persona, con una dotación de 15000 pesetas, en Bilbao para que estuviera "cerca" del Ministerio de Industria. La actuación lobista de las patronales catalanas queda constatada cuando en julio de 1939 tanto el instituto como el gremio citan en sus actas respectivas el agradecimiento $\mathrm{y}$ reconocimiento hacia los mediadores que actuaban en el ministerio

${ }^{19}$ Ibid., p. 79.

20 "Como catalanes, afirmamos que nuestra tierra quiere seguir unida a los otros pueblos de España, por el amor fraternal y por el sentimiento de la comunidad de destino que nos obliga a todos a contribuir con el máximo sacrificio a la obra común de liberalización de la tiranía roja y de preparación de la grandeza futura de España. Como catalanes, saludamos a nuestros hermanos que a millares, vencidos los obstáculos que opone la situación de Cataluña, lucha en las filas del ejército libertador y exhortamos a todos los catalanes para que tan pronto como sea materialmente posible se junten a ellos, ofrendando sus vidas para el triunfo de la causa de la civilización en la lucha contra la barbarie anarquista y comunista." Entre los firmantes estaban Joaquín M. de Nadal, abogado, ex diputado a Cortes por Barcelona; José Ballvé Pallie, fabricante, directivo de la Asociación de Fabricantes de Hilados; Pedro Cabot, presidente de la Unión de Sindicatos Agrícolas de Cataluña, de la Cambra Oficial Agrícola de Barcelona, y de la Federación de Sindicatos Agrícolas del Litoral; R. de Caralt, industrial; Enrique de Caralt, industrial; I. Baruola, industrial; R. Pujol Güell, industrial; Francisco Soler, industrial, ex diputado provincial; José de Caralt, ingeniero industrial, y Enrique y Carlos Maristany, industriales. Carta a vicepresidencia del gobierno, 9 de noviembre de 1936, en Archivo Presidencia del Gobierno (APG), leg. 8. 
(Alfonso Sala Argemí, conde de Egara, y José María Milá y Camps, conde de Montseny). El instituto es el más explícito dando las gracias por "el feliz resultado de la labor realizada al servicio de los intereses de la industria", pero el gremio lo es más en suministrarnos información al reflejar en su libro de actas la aprobación de una derrama entre los socios para hacer frente "a los enormes gastos ocasionados por las campañas realizadas para resolver el problema de la venta de nuestras manufacturas". ${ }^{21}$ De manera explícita resume la importancia de ser activos el presidente del instituto, cuando manifestó públicamente que: "no podemos estar quietos en nuestro despacho ocupándonos exclusivamente de lo nuestro, sino que hay que intervenir en lo que colectivamente interesa a nuestra industria y hay que procurar hacer valer nuestra opinión dentro de los organismos que pueden tomar trascendentales disposiciones para nuestros intereses industriales". ${ }^{2}$

Por otra parte, algunos de los industriales textiles catalanes vivían en Sevilla casi desde el comienzo de la guerra civil. Entre ellos estaban los Sedó, Caralt o Maristany los cuales participaban en el tejido industrial de la ciudad al ser propietarios de la empresa textil, La María S. A. (propiedad de Sedó y Maristany), y la empresa química Industrias Andaluzas S. A. (propiedad de Sedó). De manera especial, los hermanos Sedó tenían interés particular en la evolución de HYTASA, no en vano la familia Sedó había sido una de las pioneras en la promoción del algodón en Andalucía, durante los años del "hambre de algodón" provocada por la primera guerra mundial, cuando los industriales catalanes desembarcaron en Andalucía para abastecerse de materia prima. Además, los Sedó situaron a miembros de la familia para dirigir sus empresas sevillanas y para que los mantuvieran informados de las actuaciones que acometía HYTASA, en especial la pugna se dirimió en torno a la producción de algodón en el campo andaluz como se menciona más adelante.

Ante estas fuerzas opositoras, tuvo que ser el general Queipo el que consiguiera "para HYTASA las autorizaciones para instalar estas fábricas y para importar la maquinaria" y la franquicia de aduanas. ${ }^{23}$ Sin embargo, se fracasó en obtener las divisas para pagar la maquinaria sustentándose la negativa gubernamental en que "debido a que esta fábrica no podrá producir en un futuro muy inmediato, esta comisión se ve en la necesidad

${ }^{21}$ Acta del 13 de julio de 1939, Instituto Tecnológico de Tarrassa (en adelante ITT), y acta del 31 de agosto de 1939, Gremio de Fabricantes de Sabadell.

${ }_{22}$ Acta del 13 de enero de 1949 , en ITT.

${ }^{23}$ Orden núm. 25551 del presidente de la Junta Técnica del Estado. Pumar, Tres, 1974, pp. 84,142 . 
de comunicar a ustedes la imposibilidad de facilitarles divisas para la adquisición de la maquinaria en el extranjero". ${ }^{24}$

Aun así, con la limitación de la carencia de divisas, se procedió a la constitución de Hilaturas y Tejidos Andaluces, S. A., y en enero de 1938 comenzó la construcción de la fábrica con el ánimo de influir favorablemente en la concesión de las divisas para traer la maquinaria en el tiempo que se tardara en edificarla. ${ }^{25}$ Sin embargo, los planes se torcieron definitivamente a raíz del nombramiento del primer gobierno civil franquista que supuso la llegada al Ministerio de Industria de Juan Antonio Suanzes (febrero de 1938). ${ }^{26}$ De inmediato, el ministro comenzó a poner trabas a HYTASA buscando retrasar, e incluso impedir, la instalación y puesta en producción de la fábrica. ${ }^{27} \mathrm{El}$ argumento utilizado por el Ministerio de Industria era que los permisos que había otorgado la Junta Técnica del Estado ya no eran válidos y se debía recomenzar todo el procedimiento. Se enfrentaban dos concepciones diferentes de las necesidades de España: por un lado, Suanzes, con el propósito de financiar el esfuerzo de guerra, no preveía desviar divisas hacia las importaciones de maquinaria, pues, a su juicio, no eran prioritarias en aquellos años; por el otro, los gestores de HYTASA defendían sus intereses y consideraban como perfecto un escenario bélico que tenía apartada a la producción catalana y desabastecida a la población, situación que desaparecería en cuanto finalizara la contienda civil.

${ }^{24}$ Estado Español, Junta Técnica del Estado, Comisión de Industria, Comercio y Abastos, cítese el núm. 13217, en Archivo de la Delegación Provincial de Industria de Sevilla (en adelante ADPIS), exp. HYTASA.

${ }^{25}$ Con un gran aparato de propaganda gracias a la implicación de las principales figuras políticas, militares y religiosas de la ciudad. "Asistieron todas las autoridades: el general Queipo de Llano, el vicario general de la diócesis, don Jerónimo Armario, en representación de su eminencia; el alcalde, señor marqués de Soto Hermoso; el señor Fernández por el gobernado civil; el rector de la universidad, señor Mota Salado; todo el consejo de administración de las Hilaturas y Tejidos Andaluces, Sociedad Anónima, con su presidente don Luis Cobián y el secretario, don Prudencio Pumar; el delegado regional del Trabajo, señor Pérez de Ayala; representaciones de entidades comerciales de la ciudad, lo más representativo, en fin, de la ciudad.” El Correo de Andalucía, 15 de enero de 1938.

${ }^{26}$ De la biografía de Suanzes podemos destacar la concepción que el ministro tenía de los empresarios privados, de los que pensaba que eran como pupilos a los que había que enseñar patriotismo, pues consideraba que el capital privado era "cobarde". Intervino activamente en las comisiones reguladoras de la producción en las que se preparaban proyectos para levantar la economía de guerra, entre los planes se incluían las fibras naturales. Ya como ministro de Industria y Comercio se traslada a Bilbao y realiza la reorganización industrial del país, coordinando los abastecimientos de guerra y estableciendo las bases de protección y defensa de la industria. Era, ante todo, un defensor a ultranza de los intereses del Estado frente a grupos de presión, sobre todo si estos tenían representantes en el Consejo de Ministros. Véanse Schwartz y González, Historia, 1978; Martín y Comín, INI, 1991; Ballestero, Juan, 1993, y Comín, "Mitos”, 2000.

${ }^{27} \mathrm{La}$ animadversión del ministro Suanzes hacia HYTASA casa con su interés en reducir las importaciones de maquinaria, pero no tanto con su interés en eliminar por completo las importaciones de hilados. Véase Catalan, "Guerra", 2006, p. 208. 
El ministro adoptó una posición de fuerza que los gestores de HYTASA trataron de cambiar mediante una entrevista personal. Fue una de las figuras políticas del momento, Pedro Gamero del Castillo (gobernador civil de Sevilla y jefe provincial de Falange y posterior ministro sin cartera), quien actuó como intermediario. La entrevista -relata Pumar-fue tensa y violenta y no logró desatascar el impasse en el que vivía HYTASA ya que el ministro se negaba a autorizar las divisas y se mantenía en la necesidad de retrotraer todo el procedimiento al principio. Así, durante la primavera de 1938 prosiguió el pulso entre HYTASA y el Ministerio de Industria. En abril, los directivos de HYTASA creyeron que les corresponderían alguno de los cupos mensuales de divisas que iban a establecerse, dada la antigüedad de su solicitud (enero de 1938) y, en cambio, se les contestó que debían atenerse al oficio sobre autorización para importar maquinaria (abril de 1938). En mayo, la fábrica tenía las obras muy avanzadas y estaba casi en condiciones de empezar la producción siéndole urgente importar la maquinaria y aunque lograron que se les concedieran las divisas quedaba obtener la aprobación del ministro, que niega personalmente las divisas bajo cualquier condición. ${ }^{28}$

La oposición del ministerio se refuerza aún más a partir de septiembre de 1938, cuando la delegación de Industria de Sevilla le traslada a HYTASA los avisos para el cumplimiento de las nuevas disposiciones legales sobre tramitación de autorizaciones para las nuevas industrias y sobre las nuevas tributaciones y le comunica que "quedaba caducada la autorización para instalar la fábrica y para importar maquinaria". ${ }^{29} \mathrm{El}$ eje de los problemas será la imprescindible, y expresa, autorización del Ministerio de Industria para implantar una fábrica de nueva planta, añadiendo que cualquier tramitación, aun si hubiera sido comenzada previamente al decreto, debía someterse a las nuevas condiciones. Es decir, HYTASA se veía obligada a empezar de nuevo todo el proceso burocrático por lo que el proyecto industrial sevillano estaba abocado a la paralización y al fracaso casi seguro.

Los empresarios sevillanos no se rindieron y argumentaron que, jurídicamente, no les eran aplicables las nuevas disposiciones ya que antes de ser constituida la empresa se había solicitado y obtenido la autorización pertinente de la Junta Técnica, máximo órgano ejecutivo del Estado (equiparable a un Consejo de Ministros) y que dicha solicitud fue acompañada de la obligatoria memoria explicativa del proyecto y de las justificaciones oportunas tanto para la construcción y producción de la fábrica como

${ }^{28}$ Hilaturas, "Memoria", 1939.

${ }^{29}$ Pumar, Tres, 1974, p. 89. Las medidas legislativas invocadas fueron el decreto del 20 de agosto de 1938 del Ministerio de Industria y Comercio y la orden del 5 de septiembre del Ministerio de Hacienda, en Boletín Oficial del Estado, de los días 22 de agosto y 7 de septiembre de 1938, respectivamente. Véase Pires, Regulación, 2003, pp. 22-24. 
para la importación e instalación de maquinaria, además de anexarse los informes con el número de obreros, los presupuestos, etc. Con base en aquel consentimiento del ejecutivo se compraron los terrenos, se suscribieron las acciones, se pagaron los timbres legales y los derechos reales, además de gastos de constitución y la adquisición de la maquinaria. Sólo quedó pendiente la forma de pago y el desarrollo de las autorizaciones ya concedidas. $^{30}$

Una vez enfrentados, el Ministerio, a través de Suanzes, e HYTASA, el procedimiento burocrático quedó en una situación de estancamiento, del que no pudieron rescatar a la empresa ni tan siquiera el grupo de presión que actuaba en su favor y que englobaba a personalidades con un indudable peso específico en el régimen como eran el propio general Queipo de Llano, Pedro Parias o Ramón Carranza, ambos alcaldes, gobernadores civiles de Sevilla y líderes desde primera hora del movimiento; Pedro Gamero del Castillo, gobernador civil; Eduardo Cadenas, gobernador civil, y Joaquín Benjumea Burín.

Los gestores de HYTASA se enfrentaban a una situación en la que todos los triunfos para impedir la dotación de la fábrica los tenía el ministro Suanzes, firmante último de todos los permisos. En un último intento, el general Queipo y Benjumea Burín intervienen para desatascar el trámite burocrático consiguiendo que si se renunciaba a la franquicia de Aduanas se "reconsideraría" el tema. Aunque HYTASA aceptó la condición que ponía Suanzes para conceder los permisos, estos nunca se concedieron "pues que lo que él [Suanzes] quería era que esta fábrica no se hiciera". ${ }^{31}$

La solución del contencioso se retrasó hasta agosto de 1939 cuando accedieron al segundo gobierno de Franco políticos que se habían destacado anteriormente por su apoyo decidido a HYTASA, a la vez que Suanzes deja el Ministerio de Industria. Los nuevos ministros fueron Luis Alarcón de la Lastra (ministro de Industria), Pedro Gamero del Castillo (ministro sin cartera) y Joaquín Benjumea Burín (ministro de Agricultura). Respecto a la cuestión de los permisos, el papel crucial lo desempeñó el nuevo ministro de Industria, el general Alarcón de la Lastra, cuyas relaciones de antes de la guerra con la patronal sevillana son evidentes: dirigente de la Federación Provincial de Asociaciones y Patronales Agrarias, candidato en las elecciones de 1933 y 1936 por la Confederación Española de Derechas Autónomas, vocal de la Federación Económica de Andalucía, en la que coincidió con Agustín Vázquez Armero, Santiago Benjumea y Pedro Solís.

\footnotetext{
${ }^{30}$ Instancia al ingeniero jefe de la Delegación de Industria de Sevilla, 27 de septiembre de 1938, en ADPIS, exp. HYTASA.

${ }^{31}$ Hilaturas, "Memoria", 1940.
} 
Evidentemente, con el recién estrenado gobierno, HYTASA confiaba en que se allanaran los problemas encontrados en el anterior ejecutivo. Las expectativas se cumplieron sobradamente cuando el ministro Alarcón de la Lastra, mediante la orden del 30 de diciembre de 1939, dictaba la revocación de la orden del 12 de septiembre de 1938 y regresaba el expediente a la situación original. Seguidamente, por orden del 20 de abril de 1940, el Ministerio de Industria otorgaba la franquicia arancelaria para la importación de la maquinaria de HYTASA y ordenaba que la importación se realizase "con la urgencia que el caso requiere" y en las condiciones que se otorgaron por la Junta Técnica del Estado en 1937, aunque adaptándolas a los momentos actuales. ${ }^{32}$

La lentitud que sufrió la creación de HYTASA provocó que la tardanza en obtener las autorizaciones fuera más de dos años, muy por encima de los 4.4 meses que tardaban los permisos de instalación de nuevas industrias en 1938 y de los 6.9 meses que las nuevas industrias textiles sufrían en $1943 .^{33}$ ¿Por qué se tardó tanto? HYTASA sufrió tanto la lentitud inherente a las trabas intervencionistas como la oposición de grupos de intereses opuestos. Gestores y opositores recurrieron a actores políticos en las altas instancias del régimen franquista para lograr sus objetivos y en función del grado de éxito cosechado, por unos u otros, la empresa avanzaba o se paralizaba en su camino burocrático.

\section{CONCESIONES PÚBLICAS, BENEFICIOS PRIVADOS}

La bibliografía española dispone de trabajos que explican cómo actuaron las autoridades franquistas y los empresarios en el juego para obtener recursos y favorecer o perjudicar a terceros en distintos sectores. ${ }^{34}$ Durante el primer franquismo, el Estado fue el asignador de los recursos (suplantando al mercado) y el vigilante del cumplimiento del marco institucional, convirtiéndose las decisiones de la administración en determinantes del éxito empresarial, al favorecer a unos en detrimento de otros, al decidir a quién concedía los recursos y obligando a los empresarios a que se condujeran como buscadores de rentas políticas. Esta sección se centra en estudiar cómo una serie de decisiones políticas se inclinó a favor de unas empresas,

\footnotetext{
${ }^{32}$ Orden del 20 de abril de 1940 del Ministerio de Industria, en Boletín Oficial del Estado, 20 de abril de 1940 .

${ }^{33}$ Pires, Regulación, 2003, p. 83.

${ }^{34}$ Sin ánimo de exhaustividad se pueden consultar los trabajos de Valdaliso, "Programas", 1997; Miranda "Industria", 1994, e Industria, 1998; Puig, "Modernización", 1994; Catalan, Economía, 1995; Pires, Regulación, 2003, y "Empresarios", 2005; Llordén, "Empresas”, 1995, y Garrués, IRATI, 1997.
} 
haciendo especial hincapié en tratar de cuantificar los efectos de dichas decisiones.

Tal vez, el mayor refinamiento y sofisticación en las medidas discriminatorias se produjo en el algodón. Para promover el cultivo algodonero, el Estado configuró un marco institucional intervencionista, vigente hasta 1962, por el que se fijaban los precios desde el Ministerio de Agricultura, se establecía el sistema de concesiones algodoneras y se regulaba el mercado. ${ }^{35}$ Las concesiones algodoneras, otorgadas también por el Ministerio, recayeron en las empresas privadas que actuaban en condiciones monopsónicas de compra del algodón bruto, monopolísticas en la desmotación y aprovechamiento de los subproductos y oligopolísticas en la comercialización de la fibra. Por último, desde el gobierno se reguló un mercado algodonero en el que los agentes estaban sometidos a las disposiciones y organismos gubernamentales para la distribución de la fibra. Los efectos de la política algodonera fueron el autoabastecimiento de fibra en 1962, los desajustes en el mercado de fibra y los beneficios que acumularon las empresas concesionarias de las zonas algodoneras, sobre todo aquellas que disfrutaron de la concesión de alguna de las cuatro grandes zonas productoras de algodón. ${ }^{36}$

Una vez repasado el condicionante algodonero se muestra cómo los agentes percibieron que el algodón podía ser un buen negocio en el que había que entrar y cómo para ello se tenía que estar cerca del poder político. Un poder político que en los años 1940-1942, cuando se dibujó el mapa algodonero español, estaba dirigido por Joaquín Benjumea Burín como ministro de Agricultura; este era un propietario agrícola miembro de una importante familia agrarista de Sevilla, quien desarrolló desde muy temprano una amplia labor política en puestos de responsabilidad (alcalde de Sevilla, jefe del Servicio de Regiones Devastadas, director del Instituto de Crédito Nacional) con anterioridad a las dos carteras ministeriales que desempeñó, Agricultura y Hacienda, y a la gobernación del Banco de España. Los precedentes al sistema algodonero implantado por el Ministerio podrían encontrarse en la forma de cómo se organizó la introducción del algodón y la remolacha azucarera en la zona regable del bajo Guadalqui-

${ }^{35}$ Ley de Fomento de la Producción de Plantas Textiles de 1940, desarrollada por orden del Ministerio de Agricultura, del 21 de diciembre de 1940 de concesiones provisionales. Ley de Jefatura del Estado del 29 de marzo de 1941 que modifica la denominación del Instituto de Fomento de las Plantas Textiles por el de Instituto de Fomento de la Producción de Plantas Textiles. Decreto del Ministerio de Agricultura del 29 de marzo de 1941 que organiza el citado instituto y el reglamento de 1942 que lo reorganiza.

${ }_{36}$ Maluquer, Política, 1976, pp. 78 y ss., y Fernández, “Sector”, 2002. 
vir cuando se le concedió a la empresa Bética, S. A., que construyera las fábricas precisas, la exclusividad en el territorio. ${ }^{37}$

En 1940, varios actores pugnaban por entrar en el entramado de las concesiones algodoneras. Un primer grupo se conformó en torno a los industriales que ya habían participado en la promoción del algodón en Andalucía como miembros de la Asociación Catalana para el Fomento Agrícola Algodonero (1918) que se había convertido, tras una ampliación de capital para ampliar el radio de acción de sus actividades, en la Catalana Agrícola Algodonera, S. A. (1919). Sin embrago, ante las reticencias encontradas en los medios rurales, se le cambió el nombre por el de Asociación Agrícola Algodonera Española (1920). ${ }^{38}$ Entre aquellos pioneros catalanes del cultivo del algodón en Andalucía destacaron Francisco Sans, presidente de La Catalana Agrícola Cotonera, y los dos principales impulsores de dicha sociedad Cambó, ministro de Fomento, y Luis Sedó, gobernador del Banco de España. ${ }^{39}$ Los herederos de aquellas sociedades, ya en la década de 1940, fueron los que encabezaron el grupo de empresarios que constituyeron la Compañía Española Productora de Algodón S. A. (CEPANSA), la cual monta sus propias hilaturas para cumplir con la obligación legal de concurrir a una concesión algodonera disponiendo, al menos, con la fase del hilado en la empresa. Entre los accionistas estaban Miguel Sans Mora, hijo de Francisco Sans; Juan Guitart Calva y Salvador Guardiola, este último gran propietario de tierras en la Baja Andalucía y accionista de HYTASA; acompañados de Tomas Rosés Ibotson, de la Algodonera Tomas Rosés, y José María Soldevila Grau, de Manufacturas Soldevila S. A. -ambos habían sido socios de La Catalana. También participaron José María Pujadas Maresch, de la empresa Estabell y Pahisa, S. A., y José Balcells Morato, de Fabril y Comercial Balcells e Hijos de Francisco Sans. Con una vinculación menos estrecha estaban José María Caralt, de Hilaturas Caralt-Pérez, S. A., Calcetería Hispánica y Maristany Fabril Textil, y José María Juncadella Burés de Industrias Burés S. A.

Un segundo aspirante para una concesión algodonera era la familia Bertrand, a la que Cabana señala como el "industrial algodonero individual más importante del mundo". ${ }^{40}$ Esta familia era propietaria de Textiles Reunidas, en Cádiz. Un tercer grupo era la familia Sedó que, separada del grupo constituido en CEPANSA, intentaba sola alcanzar una concesión. La familia Sedó había sido pionera del algodón en Andalucía, y disponía de

${ }^{37}$ Resulta interesante que, aunque no aparece como inversor en la sociedad, el ministro Joaquín Benjumea Burín fuera uno de los principales propietarios de la zona regable del Bajo Guadalquivir (313 hectáreas).

${ }^{38}$ Fernández, "Sector", 2002.

${ }^{39}$ Cabana, Fàbriques, 1993, pp. 445 y ss.

${ }^{40}$ Ibid., p. 185. 
hilaturas algodoneras en el territorio ya que utilizaba como trampolín la pequeña fábrica que habían adquirido durante la guerra civil: La María, S. A. Por último, el cuarto actor en este proceso era HYTASA.

CEPansa y Textiles Reunidas no competían ni entre ellas ni con las demás al estar radicadas en los términos de la primera y tercera zona algodonera respectivamente. La pugna se centró en torno a la segunda zona algodonera entre dos grupos empresariales: por un lado el de la familia Sedó, y por el otro HYTASA. ¿Cómo terminó la pugna? Ambos contendientes vigilaban de cerca los movimientos del otro. Así, los hermanos Sedó, ya de regreso en Barcelona, estaban completamente informados de cómo discurrían los acontecimientos, pues habían dejado en Sevilla a algunos primos como directores de sus empresas, La María e Industrias Andaluzas. Así, en octubre de 1941, los Sedó recibieron en Barcelona la siguiente carta:

En las Marismas, HYTASA tiene preparadas unas 200 ha. para cultivarlas el año próximo y no sé concretamente si ellos ofrecieron, o el señor Rosales les solicitó 100 ha. De esas preparadas para su cultivo, no sé cómo habrán quedado, aunque esto no tendría más vigencia que la de un año [se refiere a la concesión provisional por una campaña previa a la definitiva]. Parece que el cultivo le interesa, pero tropieza con el escollo de la organización que han dividido en cuatro o cinco zonas adjudicadas a otras tantas casas. ${ }^{41}$

En frente, HYTASA utilizó sus bazas políticas para alcanzar el objetivo de la concesión. Apoyando a HYTASA, pues eran accionistas de la empresa, encontramos a algunos de los miembros de las familias más influyentes del mundo político y agrario de la provincia de Sevilla, entre estas, Lasso de la Vega ${ }^{42}$ Solís, ${ }^{43}$ Benjumea, Sánchez-Ibargüen, Ostos, Armero, Candau, Guardiola, Montero de Espinosa, Medina Vilallonga, emparentados por vía matrimonial con los Fernández de Córdoba, ${ }^{44}$ Domínguez y Pérez de Vargas ${ }^{45}$ Turmo, emparentados con los Benjumea, y Murube. De forma mayoritaria, las propiedades de estas familias estaban nucleadas alrededor de los términos municipales de Carmona, Écija, Fuentes de Andalucía, La Campana, Marchena, la zona de la Vega de Sevilla, Utrera, El Coronil,

${ }^{41}$ Arxiu Nacional de Catalunya (en adelante ANC), Empresas, Industrias Andaluzas, 1013.1.

${ }^{42}$ Reunían los títulos de marquesado de Torres de la Pressa, condado de casa Galindo, condado de Peñaflor de Argamasilla y marquesado de Gómez de Barreda. Miguel Ibarra y Lasso de la Vega fue alcalde de Sevilla entre diciembre de 1940 y octubre de 1943.

${ }^{43}$ Reunían los títulos del marquesado de la Motilla y marquesado de Albentos.

${ }^{44}$ Los Fernández de Córdoba son duques de Medinaceli. Rafael Medina Vilallonga, duque de Alcalá fue alcalde de Sevilla entre octubre de 1943 y octubre de 1947.

${ }^{45}$ Jerónimo Domínguez y Pérez de Vargas, marqués del Contadero, ejerció como alcalde de Sevilla entre febrero de 1952 y septiembre de 1958. 
Montellano y Morón de la Frontera, los cuales coincidirán con lo que será el centro fundamental del cultivo del algodón en la provincia de Sevilla. ${ }^{46}$

En junio de 1942 el Ministerio de Agricultura confirma de manera definitiva las adjudicaciones de las zonas: la primera, para Textiles Reunidas, de la familia Bertrand; la segunda, para HYTASA, y la tercera zona queda adscrita a CEPANSA. Al año siguiente se le concederá a esta última una nueva zona, la cuarta. Así, en 1943, el mapa algodonero estaba prácticamente cerrado, pues entre las cuatro zonas repartidas se produjo 85\%, aproximadamente, de todo el algodón español. ${ }^{47} \mathrm{Y}$ aún más: como sólo ellas podían desmotar el algodón para obtener la fibra y aunque la legislación establecía que la tenían que repartir con los agricultores y con el Sindicato Vertical del Textil, sus hilaturas y tejedurías nunca estaban carentes de materia prima y la que les sobraba, aquella que eran incapaces de transformar en sus factorías, la vendían en un mercado nacional calificable como "hambriento de fibra" ${ }^{48}$ La escasez de materia prima era tan acuciante que, como admitía el gobernador civil de Barcelona, los empresarios debían recurrir sin dudarlo al estraperlo para proveerse de los inputs básicos: "si los industriales, que no pueden disponer de las cuotas esenciales en el momento oportuno, aceptan pasivamente esa situación, en lugar de obtener las materias primas por cualquier medio a su alcance, veríamos a miles y miles de obreros en paro por las calles a causa de los cierres temporales o de la bancarrota". ${ }^{49}$

Llegados a este punto, ¿fueron cruciales los beneficios obtenidos por las empresas de la concesión discrecional que el gobierno efectuó para cada zona algodonera? El artículo se centra en la empresa en la que más claramente participaron factores políticos al momento de concederle una zona algodonera y de la que, además, se dispone de datos suficientes para analizarla, con la limitación de que los utilizados en el artículo provienen de sus balances y su nivel de agregación impide deslindar la incidencia de la desmotación en las otras secciones que integraban la empresa (fábricas algodoneras, fábrica lanera, central térmica, detal, etc.). Aun así, es factible estimar lo que el disfrute de la zona algodonera supuso en los resultados económicos finales de la empresa sevillana.

\footnotetext{
${ }^{46}$ Patrimonio en hectáreas de algunas de las familias accionistas de HYTASA: Lasso de la Vega, 6586 ha; Solís, 5549 ha; Benjumea, 10404 ha; Sánchez-Ibargüen, 8860 ha; Ostos, 5593 ha; Armero, 4979 ha; Candau, 3846 ha; Guardiola, 1744 ha; Montero de Espinosa, 1097 ha, y Medina Vilallonga, 8887 ha. Véase Florencio, Empresariado, 1994, pp. 66-75.

${ }^{47}$ Fernández, "Sector", 2002.

${ }^{48} \mathrm{El}$ algodón en rama se llegó a pagar hasta seis veces por encima de su precio oficial. Catalan, Economía, 1995, p. 246.

${ }^{49}$ Barba, Dos, 1948.
} 
El primer paso es enmarcar a HYTASA dentro del sector textil algodonero español y para ello se utiliza la rentabilidad de los recursos propios, capital más reservas, de HYTASA y del sector algodonero nacional. La elección de esta ratio se debe a que el objetivo de esta sección es cuantificar los beneficios obtenidos, por unos determinados particulares, de las decisiones tomadas por la administración. La gráfica 1 sitúa con claridad a HYTASA por debajo de la media del sector durante todo el primer franquismo. ${ }^{50}$ El sector textil algodonero en la década de 1940 y los primeros años de la década de 1950 se mueve en una horquilla de rentabilidades situadas entre 11 y $15 \%$, que son unos datos excelentes, mientras que HYTASA dificilmente logra moverse entre 4 y $8 \%$. Sin embargo, a partir de 1955 la rentabilidad de los beneficios de la empresa sevillana comienza a crecer hasta estabilizarse por encima de la media del sector. ¿Qué podría haber motivado tan brusco y radical cambio en los resultados de HYTASA?

El motivo que está detrás de la mejora de la rentabilidad de HYTASA es el éxito del cultivo algodonero. Las zonas algodoneras se habían organizado de manera que la entidad concesionaria compraba en régimen de monopsonio todo el algodón cosechado a los agricultores, lo desmotaba en régimen de monopolio y, variando con el tiempo, lo vendía al Sindicato Nacional Textil o en el mercado de algodón de Barcelona. Esta era la gran fuente de ingresos de HYTASA (véase gráfica 2) y se comprueba comparando la rentabilidad de HYTASA con la cosecha de algodón y ambas, prácticamente, dibujan la misma curva. A partir de 1962, el sistema de concesionarias desaparece y a la par surge un buen número de cooperativas algodoneras que entran en competencia con las antiguas concesionarias, y si le sumamos el hecho de que el gobierno empezó a dejar de apoyar decididamente al algodón nacional, los resultados son evidentes: la rentabilidad de HYTASA cae irremediablemente.

$\dot{i}$ Tan elevados eran los beneficios que otorgaba el sistema algodonero a las empresas que llegaba a influir de manera determinante en la rentabilidad? En su momento, Tamames calificó a la desmotación como "el mejor negocio de la posguerra" ${ }^{51}$ En un intento de acercamiento a la realidad en la que vivió el sector desmotador se han estimado los posibles beneficios

\footnotetext{
${ }^{50}$ Las rentabilidades han precisado de uniformar los estados de balances de todas las empresas, incluyendo las amortizaciones en el inmovilizado como negativas, y practicar una adición simple, siguiendo la metodología de Tafunell. El agregado de beneficios de la industria algodonera española está basado en los beneficios netos distribuibles después de impuestos, puesto que no dispongo de todas las amortizaciones. Las empresas incluidas en la media son La Espanya Industrial, Hilaturas Fabra y Coats e HYTASA, en Anuario Financiero, 1939-1974, y Anuario Financiero y de Sociedades Anónimas, 1939-1974, e Hilaturas Forcada, Sedó, S. A., Manufacturas Burés, Manufacturas Valls y Manufacturas Viladomiu, en ANC, Balances, y en Arxiu Històric Comarcal de Manresa (AHCM), Colonia Güell.

${ }^{51}$ Tamames, Estructura, 1991, p. 156.
} 


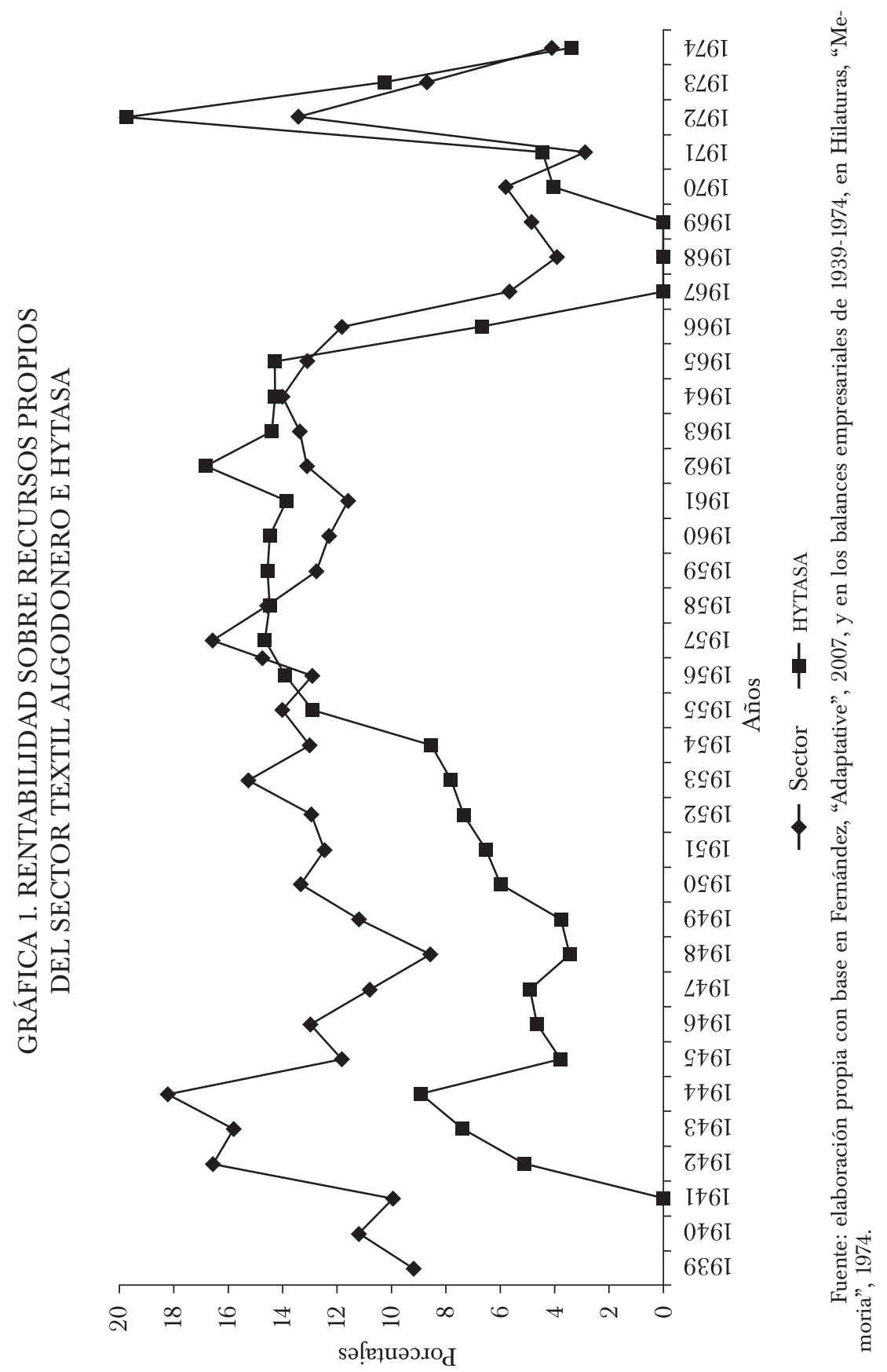



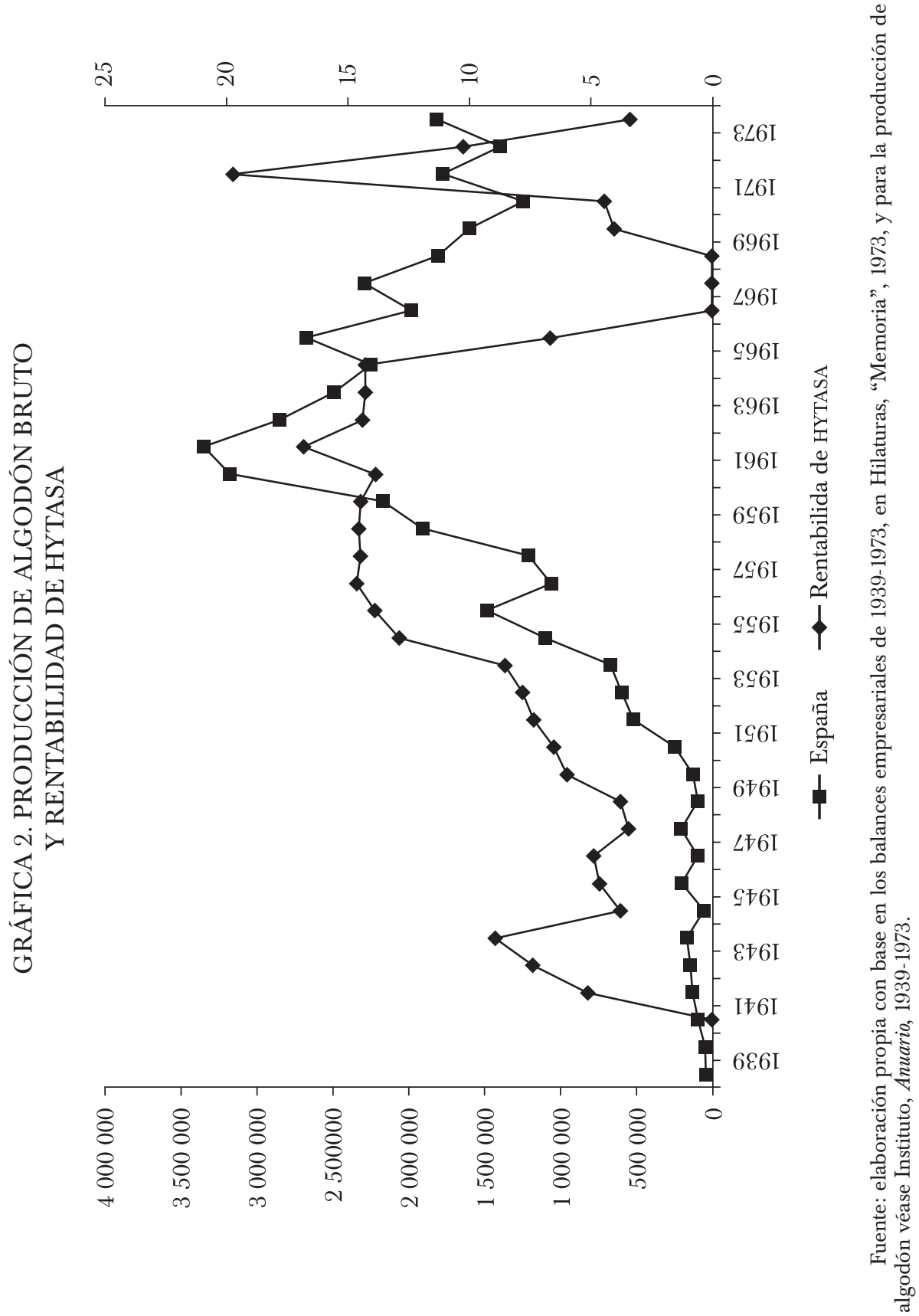
desmotadores de HYTASA y CEPANSA, como hiciera Maluquer recurriendo a los cálculos del Servicio Comercial de la Industria Textil Algodonera, aplicando la fórmula que establece el decreto de 1958. Un problema fue contar con los recursos generados y no con los beneficios líquidos, solventándolo con la aplicación, de manera inversa, del coeficiente calculado por Tafunell a los beneficios brutos. ${ }^{52}$

La dificultad del estudio contable de la desmotación radica, en primer lugar, en que no disponemos de las contabilidades completas de ninguna de las concesionarias. Un segundo problema es que las concesionarias estaban obligadas a ser desmotadoras e hiladoras -como mínimo, varias incorporaron también la producción de tejidos, por lo que en sus balances finales se agregan los resultados de las diferentes secciones. Para explicar los bajos beneficios que publicaba CEPANSA, alejados de los aquí estimados, se puede pensar que alterara los datos publicados o que vendiera a precios preferentes parte de la fibra a las empresas industriales de las que eran propietarios sus principales accionistas. La primera posibilidad es complicada y arriesgada, siendo lo más probable que los principales accionistas de CEPANSA compraran a precios preferentes la fibra para abastecer a sus fábricas.

Si se comparan los beneficios estimados para HYTASA, sólo de la desmotación de algodón, con los publicados por la empresa para el conjunto de su actividad no deja de llamar la atención que los beneficios desmotadores, en tres ejercicios, dupliquen los globales de esta. Es decir, la actividad desmotadora es la que sustenta a HYTASA, que debía acumular fuertes pérdidas en sus otras secciones, pérdidas que la empresa reconoce para sus fábricas laneras. Desaparecida la posición de privilegio, a partir de 1962, en el control de la concesión algodonera, los beneficios y rentabilidades de HYTASA se desploman.

\section{CONCLUSIONES}

Si regresamos a la propuesta de Vitelli sobre el papel que desempeñaban en la corrupción los instrumentos de la política económica, la empresa HYTASA es un magnífico ejemplo de cómo los actores interesados en frenar la iniciativa empresarial sevillana emplearon discrecionalmente la legislación industrial y también ejemplifica el caso contrario: cómo los agentes favorables a esta empresa modificaron convenientemente el marco jurídico para determinar su viabilidad. Ni unos ni otros entraron en ningún momento en los datos económicos de la empresa para determinar su via-

${ }^{52}$ Véase Tafunell, "Beneficios”, 1996, y "Beneficios”, 1998. 
Am. Lat. Hist. Econ., año 19, núm. 2 (38), mayo-agosto, 2012, pp. 193-218

\section{CUADRO 1. BENEFICIOS BRUTOS Y LÍQUIDOS DE CEPANSA E HYTASA (EN PESETAS)}

\begin{tabular}{lrrrr}
\hline & $1957-1958$ & $1958-1959$ & $1959-1960$ & 1960-1961 \\
CEPANSA & & & & \\
Beneficios brutos & 139869917 & 131690754 & 160088469 & 141049045 \\
Beneficios líquidos & 79925667 & 75251860 & 91479125 & 80599454 \\
& & & & \\
HYTASA & 8756904 & 52308973 & 63305810 & 68942069 \\
$\begin{array}{l}\text { Beneficios brutos } \\
\text { Beneficios líquidos }\end{array}$ & 5003945 & 29890841 & 36174749 & 39395468 \\
Beneficios totales & 15492629 & 15726455 & 15792180 & 18780508 \\
\hline
\end{tabular}

Fuente: elaboración propia con base en Servicio Comercial de la Industria Textil Algodonera y Anuario, 1939-1974.

bilidad o su imposibilidad. La empresa fue el terreno de juego en el que colisionaron grupos con claros intereses económicos y con la suficiente capacidad de influencia en el régimen para decantar la balanza a su favor. $\mathrm{Al}$ final, la decisión sobre HYTASA fue netamente política y apoyada en un cambio de relación en las fuerzas participantes, es decir, pasó de enfrentarse al ministro de Industria -Suanzes- a verse favorecida por tres ministros sevillanos en el gabinete.

Tanto los favores recibidos por HYTASA como los ataques hacia ella dirigidos, desde el entramado del régimen, se perciben como casos de corrupción al entenderse cómo el otorgamiento de una ayuda, o ataque, a una empresa de manera discriminatoria hacia las otras, sin que conste que mediaran pagos en dinero entre los actores. Seguramente las relaciones entre los protagonistas eran demasiado fuertes como para necesitarse la "compra" de voluntades. HYTASA, por lo tanto, es un ejemplo, sintomático, del funcionamiento del régimen franquista que demuestra la importancia de las decisiones políticas sobre las motivaciones económicas. En palabras de Andag estamos ante la ruptura de la moral pública del régimen.

La segunda parte del artículo demuestra el tráfico de influencias nacido dentro del propio aparato del Estado, cuando un ministro reorganiza el cultivo del algodón mediante concesiones de zonas algodoneras y decide quiénes son los receptores de dichas concesiones. En primer lugar, se trató de decisiones políticas que generaron enormes beneficios privados $\mathrm{y}$, en segundo lugar, de claros agravios comparativos a todos aquellos que no pudieron entrar en el negocio algodonero. 


\section{FUENTES CONSULTADAS}

\section{Archivos}

ADPIS Archivo de la Delegación Provincial de Industria de Sevilla, España.

AHCM Arxiu Històric Comarcal de Manresa, Cataluña.

ANC Arxiu Nacional de Catalunya, España.

APG Archivo Presidencia del Gobierno, España.

ITT Instituto Tecnológico de Tarrassa, Cataluña.

\section{Hemerografia}

El Correo de Andalucía, Sevilla, España.

Boletín Oficial del Estado, España.

\section{Bibliografía}

Allende Portillo, Fermín, Los empresarios vizcaínos y la política económica: industria, comercio y navegación (1886-1975), Madrid, Fundación Empresa Pública, 1995.

Alonso Hierro, Juan y Eugenio Torres Villanueva, "Funciones empresariales y desarrollo económico", Economistas, Colegio de Economistas de Madrid, año 13, núm. 63, 1995, pp. 122-129.

Andvig, Jens Christopher y Odd-Helge FJeldstad, "Research on Corruption. A Policy Oriented Survey", documento de trabajo, Noruega, 2000.

Anuario financiero, Bilbao, Banco de Vizcaya, 1939-1974.

Anuario financiero y de Sociedades Anónimas, Madrid, Editorial Sopec, 1939-1974.

Ballestero, Alfonso, Juan Antonio Suanzes, 1891-1977, Madrid, Lid, 1993.

Barba Hernández, Bartolomé, Dos años al frente del gobierno civil de Barcelona y varios ensayos, Madrid, Javier Morata, 1948.

Barciela López, Carlos Fernando, "Franquismo y corrupción económica”, Historia Social, Universidad Nacional de Educación a Distancia, núm. 30, 1998, pp. 83-96. (ed.), Autarquía y mercado negro, Barcelona, Crítica, 2003. et al., La España de Franco, 1939-1975. Economía, Madrid, Síntesis, 2001.

Braña Pinto, Francisco Javier, Mikel Buesa Blanco y José Molero Zayas, "El fin de la etapa nacionalista: industrialización y dependencia en España, 19511959”, Investigaciones Económicas, núm. 9, 1979, pp. 151-207. El Estado y el cambio tecnológico en la industrialización tardía, Madrid, Fondo de Cultura Económica, 1984.

Buesa Blanco, Mikel, "Restricciones a la libertad de industria en la política industrial española (1938 1963)”, Información Comercial Española, ICE. Revista de Economía, 
Ministerio de Industria, Turismo y Comercio-Gobierno de España, núm. 606, febrero de 1984, pp. 107-122.

Cabana, Francesc, Fàbriques i empresaris, Barcelona, Enciclopedia Catalana, 1993.

Cabrera Calvo-Sotelo, Mercedes, "Los intereses económicos organizados en España: un siglo en la historia del asociacionismo empresarial” en PABLO MARTíN Aceña y Francisco Comín Comín, La empresa en la historia de España, Madrid, Civitas, 1996, pp. 442-456.

y Fernando del Rey Reguillo, "Entre la condena y el olvido. Los empresarios y sus organizaciones en la historiografía española” en JUAN HERNÁNDEZ Andreu y José Luis García Ruiz (coord.), Lecturas de historia empresarial, Madrid, Civitas, 1994, pp. 51-74.

Carreras, Albert y Xavier Tafunell, "La gran empresa en España (1917-1974). Una primera aproximación", Revista de Historia Industrial, Universidad de Barcelona, núm. 3, 1993, pp. 127-176.

CATAlan, Jordi, La economía española y la segunda guerra mundial, Barcelona, Ariel, 1995. "Guerra e industria en las dos Españas, 1936-1939" en PABlo Martín Aceña y Elena Martínez Ruiz, La economía de la guerra civil, Madrid, Marcial Pons, 2006, pp. 161-228.

Clavera Monjonell, Joan, De la autarquía a la estabilización (1939-1959), Madrid, Edicusa, 1973.

Comín Comín, Francisco, "Estado y crecimiento económico en España: lecciones de la historia”, Papeles de Economía Española, núm. 57, 1993, pp. 32-56.

La empresa pública en la España contemporánea: formas históricas de organización y gestión (1770-1995), Madrid, Fundación Empresa Pública, 1995.

"La empresa pública en la España contemporánea: formas históricas de organización y gestión" en Francisco Comín Comín y PABlo Martín ACEÑa (eds.), La empresa en la historia de España, Madrid, Civitas, 1996, pp. 349-368.

Los rasgos históricos de las empresas en España: un panorama, Madrid, Fundación Empresa Pública, 1996.

"Los mitos y los milagros de Suanzes: la empresa privada y el INI durante la autarquía", Revista de Historia Industrial, Universidad de Barcelona, núm. 18, 2000, pp. 221-248.

"El Estado, la rigidez de los mercados y la convergencia en el siglo XX", Historia y Política: Ideas, Procesos y Movimientos Sociales, Universidad Complutense/ Universidad Nacional de Educación a Distancia, Centro de Estudios Políticos y Constitucionales, núm. 9, 2003, pp. 41-69.

y Pablo Martín Aceña, "La historia empresarial en España”, Economistas, Colegio de Economistas de Madrid, núm. 73, 1997, pp. 16-24.

Donges, Juergen Bernhard, La industrialización en España, Barcelona, Oikos Tau, 1976.

Echeberría, Goio y José Luis Herrero Castro, "La evolución de la economía española durante el periodo 1940-1988 a partir de un indicador de la tasa de 
beneficio del sector industrial”, Información Comercial Española, ICE. Revista de Economía, Ministerio de Industria, Turismo y Comercio-Gobierno de España, núm. 665, enero de 1989, pp. 9-23.

FERnÁNDEZ RocA, Francisco JaVIER, "El sector agroindustrial del algodón en España: cultivo, desmotación y Estado (1920- 1970)", Revista de Historia Industrial, Universidad de Barcelona, núm. 21, 2002, pp. 89-122.

, "The Adaptative Strategies of Spanish Cotton Industry Companies, 19391970”, Business History, Taylor and Francis Journals, vol. 49, núm. 1, 2007, pp. 75-97.

Florencio Puntas, Antonio, Empresariado agrícola y cambio económico, 1880-1936, Sevilla, Diputación de Sevilla, 1994.

Gamir Casares, Luis, Política económica de España, Madrid, Alianza Universidad, 1980. García Delgado, José Luis, “Notas sobre el intervencionismo económico del primer franquismo", Revista de Historia Económica, año III, núm. 1, 1985, pp. 135-145.

"La industrialización y el desarrollo económico de España durante el franquismo" en Jordi Nadal, Albert Carreras i Odriozola y Carles Sudrià i TRIAY, La economía española en el siglo XX. Una perspectiva histórica, Barcelona, Ariel, 1987, pp. 164-189.

GARRUÉS IRURZUN, JOSÉ, El IRATI, compañía general de maderas, fuerzas hidráulicas y tranvía eléctrico de Navarra: una empresa autoproductora comercial de electricidad, 1904-1961, Madrid, Fundación Empresa Pública, 1997.

Gómez Mendoza, Antonio, De mitos y milagros. El Instituto Nacional de Autarquía 1941 1963, Barcelona, Universitat de Barcelona, 2000.

GonzÁlez GonzÁlez, MANuel Jesús, La economía política del franquismo (1940-1970), Madrid, Tecnos, 1979.

"La autarquía económica bajo el régimen del general Franco: una visión desde la teoría de los derechos de propiedad”, Información Comercial Española, ICE. Revista de Economía, Ministerio de Industria, Turismo y Comercio-Gobierno de España, núms. 676-677, 1989-1990, pp. 19-40.

Hilaturas y Tejidos Andaluces, S. A. (HYTASA), "Memoria de la Junta General de Accionistas", ejercicio de 1938, Sevilla, 1939.

"Memoria de la Junta General de Accionistas", ejercicio de 1939, Sevilla, 1940.

"Memoria de la Junta General de Accionistas", ejercicio de 1972, Sevilla, 1973. 1974.

Instituto Nacional de Estadística, Anuario estadístico, Madrid, Instituto Nacional de Estadística, años 1939-1973.

Llordén MiÑAmbres, Moisés, "Empresas y empresarios de promoción y construcción inmobiliaria en Asturias. El ejemplo de Gijón” en MoISÉs LLORdÉn MiÑAMBRES (comp.), De empresas y empresarios en la España contemporánea, Oviedo, Universidad de Oviedo, 1995, pp. 135-177. 
MaluQuer i Sostres, JoAQuim, La política algodonera 1940-1970, Barcelona, Servicios de Estudios de Banca Catalana, 1976.

Martín Aceña, Pablo y Francisco Comín Comín, INI. 50 años de industrialización en España, Madrid, Espasa Calpe, 1991.

Miranda EnCARnación, José ANTONiO, "La industria del calzado española en la posguerra: los efectos del intervencionismo sobre una industria de bienes de consumo", Revista de Historia Económica. Journal of Iberian and Latin American Economic History, Instituto Figuerola de Historia Económica-Universidad Carlos III de Madrid, año 12, núm. 2, 1994, pp. 317-339.

, La industria del calzado en España, Alicante, Generalitat Valenciana, 1998. "El fracaso de la industrialización autárquica" en CARLOS FERNANDO BARCIEla López (ed.), Autarquía y mercado negro, Barcelona, Crítica, 2003, pp. 95-121.

Myro, RAFAEL, "La industria, de la autarquía a la integración en la CE" en José LUIS García Delgado, España, economía, Madrid, Espasa Calpe, 1993, pp. 197-230.

Pires Jiménez, Luis EduARdo, Regulación industrial y atraso económico en la dictadura de Franco, Madrid, Dykinson/Universidad Rey Juan Carlos, 2003.

"Los empresarios y el Estado en torno a las intervenciones del régimen de Franco: la regulación de la inversión industrial", Investigaciones de Historia Económica. Revista de la Asociación Española de Historia Económica, núm. 2, 2005, pp. 145-180.

PUIG RAPOSO, NÙRIA, "Modernización y regulación. La industria alcoholera española, 1856-1953" en JORDI NADAL y JORDI CATALAN (coords.), La cara oculta de la industrialización española: la modernización de los sectores no líderes (siglos XIX y XX), Madrid, Alianza Universidad, 1994, pp. 99-126.

Pumar, Prudencio, Tres personalidades influyentes en mi vida, Sevilla, Editorial Prensa Española, 1974.

SAutu, Ruth, Catálogo de prácticas corruptas. Corrupción, confianza y democracia, Buenos Aires, Lumiere, 2004.

Schwartz, Pedro y Manuel Jesús González, Una historia del Instituto Nacional de Industria, Madrid, Tecnos, 1978.

TAFunell, XAVier, "Los beneficios empresariales en España, 1880-1981: elaboración de una serie anual", documento de trabajo núm. 9601, Madrid, Fundación Empresa Pública-Programa de Historia Económica, 1996.

"Los beneficios empresariales en España, 1880-1981", Revista de Historia Económica. Journal of Iberian and Latin American Economic History, Instituto Figuerola de Historia Económica-Universidad Carlos III de Madrid, núm. 3, 1998, pp. 707-746.

Tamames, Ramón, La república. La era de Franco, Madrid, Alianza Universidad, 1973. Estructura económica de España, Madrid, Alianza, 1991.

Torres Villanueva, Eugenio, "El empresario" en José Luis García Delgado, Lecciones de economía española, Madrid, Civitas, 1996, pp. 173-187. 
, "Funciones empresariales y desarrollo económico" en SANTiago M. LÓPEZ García y Jesús MARÍA VALDALISO GaGO, ¿Qué inventen ellos?: tecnología, empresa y cambio económico en la España contemporánea, Madrid, Alianza, 1997, pp. 53-84. "La empresa en la autarquía, 1939-1959. Iniciativa pública versus iniciativa privada" en CARlos Fernando BARCIELA LóPEZ, Autarquía y mercado negro: el fracaso económico del primer franquismo, 1939-1959, Barcelona, Crítica, 2003, pp. 169-216.

, "Los empresarios: entre la revolución y la colaboración” en PABLO MARtín Aceña y Elena Martínez Ruiz, Madrid, Marcial Pons, 2006, pp. 431-460.

VAldaliso Gago, Jesús María, "Programas navales y desarrollo económico: la empresa nacional 'Elcano' de la marina mercante y el sueño industrializador de Suanzes (1942-1963)", Revista de Historia Industrial, Universidad de Barcelona, núm. 12, 1997, pp. 147-177.

Vitelli, Guillermo, La corrupción como producto de los procesos históricos y de las lógicas de las estructuras político-económicas, México, Congreso Latinoamericano de Historia Económica, 2010. 